\title{
Aspectos clínicos e epidemiológicos dos idosos com febre de Chikungunya
}

\author{
Clinical and epidemiological aspects of elderly patients with Chikungunya fever
}

Cynthia Angélica Ramos Oliveira Dourado ${ }^{1,2}$, Evelyn Maria Braga Quirino ${ }^{1}$, Clarissa Mourão Pinho ${ }^{1}$, Mônica Alice Santos da Silva ${ }^{1}$, Slayne Rayane Gomes de Souza ${ }^{2}$, Maria Sandra Andrade ${ }^{1}$

Objetivos: caracterizar os aspectos clínicos e epidemiológicos dos idosos acometidos pela febre de Chikungunya. Métodos: estudo transversal e retrospectivo de casos confirmados de febre de Chikungunya em idosos. Para as variáveis categóricas foram calculadas as frequências e percentuais. Utilizou-se o teste qui-quadro para comparação de proporções das distribuições das variáveis intragrupos. Resultados: foram avaliados 300 idosos com predominância de mulheres (63,0\%), idade média de 70,49 anos, cores parda e preta (92,0\%), moradores da zona urbana (95,3\%), notificados na fase crônica da doença $(57,3 \%)$, que necessitaram de hospitalização $(74,3 \%)$ e com comorbidades $(63,0 \%)$. Os sintomas mais prevalentes foram febre e artralgia $(100,0 \%)$, cefaleia $(98,0 \%)$ e lombalgias (96,3\%). Conclusão: os casos de febre de Chikungunya tendem a ser mais graves em pacientes idosos e com maiores repercussões clínicas, em especial com a presença de sintomas álgicos.

Descritores: Febre de Chikungunya; Idoso; Epidemiologia; Enfermagem em Saúde Pública.

Objectives: to characterize the clinical and epidemiological aspects of elderly people affected by Chikungunya fever. Methods: cross-sectional and retrospective study of confirmed cases of Chikungunya fever in elderly people. Frequencies and percentages were calculated for categorical variables. The chi-square test was used for intragroup comparisons of the proportional distribution of variables. Results: three hundred elderly were evaluated. There was a predominance of women (63.0\%), with a mean age of 70.49 years, brown and black skin color (92.0\%), living in the urban area (95.3\%), reporting the disease in the chronic phase (57.3\%), requiring hospitalization $(74.3 \%)$, and with comorbidities (63.0\%). The most prevalent symptoms were fever and arthralgia (100.0\%), headache (98.0\%), and low back pain (96.3\%). Conclusion: cases of Chikungunya fever tend to be more severe in elderly patients and have greater clinical repercussions, especially with the presence of symptoms of pain.

Descriptors: Chikungunya Fever; Aged; Epidemiology; Public Health Nursing.

\footnotetext{
${ }^{1}$ Universidade de Pernambuco. Recife, PE, Brasil.

${ }^{2}$ Centro Universitário Estácio do Recife. Recife, PE, Brasil.

Autor correspondente: Maria Sandra Andrade

Rua Arnóbio Marques, 310, Santo Amaro. CEP: 50.100-130. Recife, PE, Brasil. E-mail: sandra.andrade@upe.br
} 


\section{Introdução}

A febre de Chikungunya teve os primeiros casos confirmados no Brasil na cidade do Oiapoque, em setembro de 2014. Trata-se de um agravo de alto impacto na saúde pública registrado no Brasil em 2015. Dentre os 38.332 casos suspeitos da doença distribuídos em 696 municípios, 13.236 (34,5\%) foram confirmados e, destes, seis óbitos ocorreram em pessoas com média de idade de $75 \operatorname{anos}^{(1-2)}$.

Uma característica importante da doença são as dores articulares mesmo após o período de viremia, na fase aguda da doença. A viremia se caracteriza pela presença do vírus no sangue e dura cerca de 5 a 10 dias após o início dos sintomas, que incluem febre, dor nas costas, artralgia e dor de cabeça de início súbito e intenso $^{(1)}$. A persistência de dor articular e muscular pode durar de meses a anos, não havendo ainda consenso na literatura sobre o período médio de duração desse agravo. Estima-se que uma média de um terço das pessoas acometidas pela doença evolui para a fase crônica, caracterizada por limitação física, interferência na rotina laboral e na qualidade de vida ${ }^{(2-3)}$.

Evidências científicas demostram que a fase crônica da Chikungunya pode estar associada à evolução de doenças reumáticas inflamatórias, com o surgimento prematuro, em pessoas já predispostas, de artrite reumatoide e artrite psoriática, implicando, inclusive, a ocorrência de formas mais graves e insidiosas $^{(4)}$. A infecção pelo vírus Chikungunya (CHIKV) funciona como um fator predisponente do processo inflamatório, que se inicia logo após a inoculação do vírus, favorecendo a liberação precoce de citocinas pró-inflamatórias em vários tecidos, incluindo músculos e articulações ${ }^{(4-6)}$.

As repercussões clínicas geradas pela febre de Chikungunya impactam principalmente os idosos. Nesse grupo etário, a maior atenção é para o risco de complicações decorrentes das doenças prévias e a maior propensão de letalidade, em decorrência da baixa reserva orgânica e imunológica natural da idade, apesar da doença possuir um baixo potencial de letalidade em outros grupos etários ${ }^{(4-5)}$.
Além disso, o acréscimo da população idosa brasileira nos últimos anos veio acompanhado do aumento das doenças crônicas não comunicantes e de consequentes limitações físicas. A febre de Chikungunya também é considerada uma doença desencadeante dessas limitações. 0 acometimento dos idosos, por esse agravo, incorpora maiores repercussões funcionais e demanda mais atenção e melhor entendimento das implicações da doença nessa população ${ }^{(6-7)}$.

Assim, os cuidados em saúde a pacientes idosos acometidos pela febre de Chikungunya com doenças preexistentes e comorbidades são essenciais para manutenção da saúde e controle das repercussões clínicas decorrentes da doença. Nesse sentido, considerando a vulnerabilidade desses pacientes e as consequências que a febre de Chikungunya pode trazer a esses sujeitos, estudos a respeito dos impactos que a infecção pode causar a esse grupo que aparentemente é um dos mais afetados são muito importantes.

Nessa perspectiva, o objetivo deste estudo foi caracterizar os aspectos clínicos e epidemiológicos dos idosos acometidos pela febre de Chikungunya.

\section{Métodos}

Trata-se de estudo transversal, retrospectivo, que utilizou os dados do Sistema de Informação de Agravos de Notificação dos casos confirmados de febre de Chikungunya registrados no setor de vigilância epidemiológica da Secretaria Municipal de Saúde de Goiana, Pernambuco, no período de janeiro de 2015 a abril de 2017. Os primeiros casos confirmados, por critério laboratorial ou clínico epidemiológico, ocorreram no município de Goiana em setembro de 2015, desde então foi registrado um aumento crescente de casos, com a situação de surto caracterizada em 2016.

A população do estudo se caracterizou por todos os pacientes idosos diagnosticados e notificados com infecção pelo CHIKV no município de estudo. A seleção da amostra foi intencional. Os critérios de inclusão foram os casos notificados e confirmados para febre de Chikungunya em pacientes com idade igual ou superior a 60 anos, com e sem comorbidades as- 
sociadas. 0 critério de exclusão foram fichas de notificação que apresentaram registros incompletos das variáveis de interesse do estudo. No período selecionado para a pesquisa, foram registrados 3.064 casos confirmados por critério laboratorial, dos quais 522 ocorreram em idosos. Levando-se em consideração os critérios elencados, a amostra final foi de 300 idosos.

Elaborou-se um instrumento para a coleta de dados que serviu com roteiro para registrar as informações relacionadas ao objetivo de pesquisa pretendido. Nesse instrumento foram consideradas as variáveis do estudo que posteriormente serviram para estruturar o banco de dados para análise estatística. As variáveis foram de caracterização da pessoa, como faixa etária, sexo, raça e procedência, e as de caracterização clínica, como presença de comorbidades associadas, estágio da doença na notificação, necessidade de internação hospitalar e sinais e sintomas associados.

Os dados foram registrados com dupla entrada, pelos próprios pesquisadores, em uma planilha eletrônica de dados (Microsoft Excel囚), e transferidos para o software Statistical Package for the Social Sciences, versão 25, para realização da análise. Na avaliação das variáveis qualitativas, foram calculadas as frequências e percentuais de cada variável, como também foi realizado o teste qui-quadro $\left(\mathrm{X}^{2}\right)$ para comparação de proporções das distribuições dos resultados dentro de cada grupo. Em todos os testes foi considerado significativo quando $\mathrm{p}<0,05$.

Por utilizar dados de fonte secundária, não foi necessário o uso do Termo de Consentimento Livre e Esclarecido. 0 projeto de pesquisa foi aprovado pelo comitê de ética em pesquisa com número de Certificado de Apresentação para Apreciação Ética no 66113417.4.0000.5640 e parecer no 2.037.142.

\section{Resultados}

Os idosos avaliados $(\mathrm{n}=300)$ foram em maior número do sexo feminino, idade variando de no mínimo 60 ao máximo de 106 anos, com média de 70,5 anos e desvio padrão de $\pm 9,36$. 0 teste de comparação de proporção foi significativo para todos os fatores avaliados $(\mathrm{p}<0,05)$, indicando que o perfil sociodemográfico dos idosos e os aspectos clínicos da doença são os mais prevalentes na amostra pesquisada. Na caracterização do perfil clínico, verifica-se que a maioria dos idosos foi notificada na fase crônica da doença, com necessidade de hospitalização, e possuía comorbidades associadas (Tabela 1).

Tabela 1 - Caracterização sociodemográfica e clínica dos idosos notificados com febre de Chikungunya

\begin{tabular}{|c|c|c|}
\hline Fator avaliado & n (\%) & $\mathbf{p}^{*}$ \\
\hline \multicolumn{3}{|l|}{ Idade (anos) } \\
\hline 60 a 75 & $220(73,3)$ & \\
\hline 76 a 90 & $72(24,0)$ & $<0,001$ \\
\hline$\geq 91$ & $8(2,7)$ & \\
\hline \multicolumn{3}{|l|}{ Sexo } \\
\hline Masculino & $111(37,0)$ & $<0,001$ \\
\hline Feminino & $189(63,0)$ & \\
\hline \multicolumn{3}{|l|}{ Procedência } \\
\hline Zona Urbana & $286(95,3)$ & $<0,001$ \\
\hline Zona Rural & $14(4,7)$ & \\
\hline \multicolumn{3}{|l|}{ Raça } \\
\hline Branca & $22(7,3)$ & \\
\hline Parda & $170(56,7)$ & \\
\hline Negra & $106(35,3)$ & $<0,001$ \\
\hline Amarelo & $2(0,7)$ & \\
\hline Indígena & - & \\
\hline \multicolumn{3}{|c|}{ Estágio da doença } \\
\hline Aguda & $128(42,7)$ & 0,011 \\
\hline Crônica & $172(57,3)$ & \\
\hline \multicolumn{3}{|l|}{ Hospitalização } \\
\hline Sim & $224(74,7)$ & $<0,001$ \\
\hline Não & $76(25,3)$ & \\
\hline \multicolumn{3}{|l|}{ Comorbidades } \\
\hline Sim & $189(63,0)$ & $<0,001$ \\
\hline Não & $111(37,0)$ & \\
\hline
\end{tabular}

As comorbidades registradas com maior frequência foram hipertensão arterial (46,7\%), seguida da diabetes mellitus $(34,0 \%)$. Para todos os fatores analisados, o p-valor foi significativo, indicando maior proporção para os casos com maior percentual, exceto para a hipertensão arterial em relação à proporção de 
não hipertensos na qual a diferença não foi significativa (Tabela 2).

Tabela 2 - Comorbidades apresentadas pelos idosos notificados com febre de Chikungunya

\begin{tabular}{lccc}
\hline \multirow{2}{*}{ Fator } & Sim & Não & \multirow{2}{*}{ p* $^{*}$} \\
\cline { 2 - 3 } & $\mathbf{n ~ ( \% )}$ & $\mathbf{n ~ ( \% )}$ & \\
\hline Diabetes mellittus & $102(34,0)$ & $198(66,0)$ & $<0,001$ \\
Hipertensão & $104(46,7)$ & $160(53,3)$ & 0,248 \\
Doenças hematológicas & $4(1,3)$ & $296(98,7)$ & $<0,001$ \\
Hepatopatias & $18(6,0)$ & $282(94,0)$ & $<0,001$ \\
Doença renal crônica & $19(6,3)$ & $281(93,7)$ & $<0,001$ \\
Doença gástrica & $38(12,7)$ & $262(87,3)$ & $<0,001$ \\
Doença autoimune & $2(0,7)$ & $298(99,3)$ & $<0,001$ \\
\hline *Teste $\mathrm{X}^{2}$ & & &
\end{tabular}

Tabela 3 - Sinais e Sintomas apresentados pelos idosos notificados com febre de Chikungunya

\begin{tabular}{lccc}
\hline \multirow{2}{*}{ Fator } & $\mathbf{S i m}$ & $\mathbf{N a ̃ o}$ & \multirow{2}{*}{$\mathbf{p}^{*}$} \\
\cline { 2 - 3 } Febre & $\mathbf{n}(\mathbf{6})$ & $\mathbf{n}(\mathbf{\%})$ & \\
Mialgia & $300(100,0)$ & - & $<0,001$ \\
Cefaleia & $294(98,0)$ & $6(2,0)$ & $<0,001$ \\
Exantema & $199(66,3)$ & $101(33,7)$ & $<0,001$ \\
Vômitos & $207(69,0)$ & $93,0(31,0)$ & $<0,001$ \\
Náuseas & $241(80,3)$ & $59(19,7)$ & $<0,001$ \\
Lombalgias & $289(96,3)$ & $11(3,7)$ & $<0,001$ \\
Conjuntivite & $3(1,0)$ & $297(99,0)$ & $<0,001$ \\
Artrite & $97(32,3)$ & $203(67,7)$ & $<0,001$ \\
Artralgia & $300,0(100,0)$ & - & $<0,001$ \\
Petéquias & $4(1,3)$ & $296(98,7)$ & $<0,001$ \\
Leucopenia & - & $300(100,0)$ & $<0,001$ \\
Dor retro orbital & $6(2,0)$ & $294(98,0)$ & $<0,001$ \\
Prova do Laço & - & $300(100,0)$ & $<0,001$ \\
\hline *Teste X & & & $<0,001$ \\
\hline & & &
\end{tabular}

Considerando os sinais e sintomas, febre e artralgia foram relatadas em todos os casos. Percebe-se também um número expressivo de casos de sintomatologias álgicas de característica articular, como lombalgias 289 (96,3\%) e artralgias $300(100,0 \%)$, mes- mo com um percentual considerável de paciente que relatam ausência de doença articular 203 (67,7\%). Para todos os fatores analisados, o p-valor foi significativo indicando que em idosos os sinais e sintomas ocorrem de forma semelhante (Tabela 3).

\section{Discussão}

0 presente trabalho apresenta algumas limitações, pois os casos de febre de Chikungunya considerados foram apenas os notificados, excluindo os possíveis casos subnotificados. Observa-se que a notificação de doenças infecciosas é abaixo do esperado no Brasil. Além disso, os estudos de dados secundários limitam a análise às variáveis presentes nas fichas de notificação e não permitem explorar outros fatores determinantes e condicionantes relacionados ao desenvolvimento da doença.

Contudo, a pesquisa contribuiu com informações que permitem aos profissionais de saúde conhecer aspectos clínicos e epidemiológicos de pacientes idosos acometidos por febre de Chikungunya, inclusive no período crônico da doença, além da oportunidade de reforçar a necessidade de medidas de prevenção, de autocuidado e a busca dos serviços de saúde de forma ágil. Para os profissionais de Enfermagem, pode contribuir para o planejamento do cuidado prestado a essa população, na notificação de casos suspeitos, em especial nos atendimentos realizados nas unidades básicas de saúde.

Como se trata de uma doença com registro recente no Brasil, com diversos estudos em andamento, os resultados da presente pesquisa podem contribuir com ações estratégicas de prevenção e controle da exposição a vetores e assim prevenir a ocorrência de novos casos, além de alertar para a possibilidade de casos mais graves em pacientes idosos e com comorbidades associadas, ou doença articular preexistente.

A febre de Chikungunya constitui um problema de saúde pública emergente, principalmente devido às manifestações musculoesqueléticas agudas e de longo prazo debilitantes. Nessa perspectiva, é necessário 
planejar cuidados à saúde de acordo com as necessidades singulares dos idosos visando ao bem-estar e adotando medidas que preservem a sua autonomia. Ser independente é um valor importante para os idosos. Portanto, tornar-se dependentes de cuidados, em virtude das sequelas da febre de Chikungunya, pode ter grandes consequências físicas e emocionais para essas pessoas ${ }^{(5-8)}$.

A promoção de estratégias saudáveis de enfrentamento constitui um grande desafio, que pode ser alcançada através do acompanhamento nas unidades básicas de saúde e em visitas domiciliares realizadas pelas equipes de saúde. Estudos clínicos mostram uma grande variedade de manifestações clínicas da doença, ainda não existe um consenso abrangente sobre o quadro clínico da febre de Chikungunya. No entanto, as sequelas de longo prazo autopercebidas em uma proporção considerável de pacientes impactam significativamente a qualidade de vida. 0 sexo feminino, idade avançada, algumas comorbidades e a gravidade da fase aguda foram associadas à artralgia persistente $\mathrm{e}^{(5-6)}$.

A feminização da velhice, caracterizada pela maior participação feminina quanto mais velha for a população analisada, reflete-se neste estudo. As mulheres idosas são maioria em todas as populações do mundo e vivem de cinco a sete anos mais do que os homens na mesma faixa etária. A frequência de acometimentos ainda pode ser explicada em virtude de, habitualmente, as mulheres ficarem mais tempo na residência, onde boa parte das infecções acontece ${ }^{(9)}$.

Ser do sexo feminino, ter idade superior a 40 anos, envolvimento articular na fase aguda, diagnóstico prévio de doença articular e presença de comorbidades como diabetes mellittus foram fatores mais associados à doença, tanto na fase aguda como na fase crônica. ${ }^{(5-6)}$. No planejamento do cuidado à saúde de pacientes idosos acometidos pela febre de Chikungunya esses aspectos devem ser considerados.

A predominância da variável raça/cor parda e negra leva à reflexão sobre os determinantes sociais da saúde. Fatores étnico/raciais têm sido associados a um maior acometimento de problemas de saúde na população negra/parda. A permanência de iniquidades sociais, como a manutenção de pessoas em exclusão social, com dificuldade de acesso à saúde, renda e trabalho, perpetua condições de vulnerabilidade em grupos específicos, estando os negros na maioria das vezes em condições menos favorecidas ${ }^{(10)}$.

Doenças causadas por mosquitos atingem de forma desproporcional as populações menos favorecidas de várias formas. Morar em grandes centros urbanos, em locais sem infraestrutura e condições mínimas de saneamento básico, dificuldade de acesso aos serviços de saúde, conviver em proximidade devido à alta densidade populacional, falhas no fornecimento de água e necessidade de manter reservatórios muitas vezes malconservados são fatores que propiciam a disseminação dos mosquitos e acometimento da doença nas camadas menos favorecidas da população ${ }^{(11)}$.

Além disso, o aumento da urbanização, os movimentos migratórios e a capacidade do vírus de se adaptar para sobreviver, assim como as crises hídricas, facilitam a deposição de ovos e o desenvolvimento de mais vetores, somado ao fato de que o mosquito se adaptou bem aos ciclos urbanos de reprodução, o que favorece o acréscimo de casos da doença ${ }^{(12-13)}$. Ações de controle vetorial, educação em saúde e a mobilização da população através dos movimentos sociais e da participação nas conferências de saúde podem contribuir para melhoria das condições que propiciam o desenvolvimento de vetores. Os profissionais de saúde precisam abordar essas questões no atendimento em saúde da população, em especial, no atendimento na atenção básica.

Os sinais clínicos da infecção por arboviroses são variados em humanos. Apresentam-se com sintomas similares ao de uma gripe, com aparição de febre alta (39ㅇ a $40^{\circ} \mathrm{C}$ ) e cefaleia, enquanto a erupção cutânea e artralgia têm como evidências a poliartrite/poliartralgia e exantema maculopapular. Os indivíduos acometidos pela infecção ainda podem apresentar sintomatologia que varia de dor nas costas 
(96,4\%), mialgia $(11,2 \%)$, náuseas $(80,7 \%)$ e vômitos $(69,7 \%)^{(14)}$.

As dores nas articulações podem ser intensas e acompanhadas de febre abrupta e ocorrem em $100,0 \%$ dos enfermos, progressivamente a artrite vai se desenvolvendo em poucos dias, ocorrendo em $100,0 \%$ dos pacientes ${ }^{(14)}$. Na presente pesquisa, esse dado é consonante tendo em vista que a lombalgia e a artralgia se apresentaram relevantes diante das outras manifestações clínicas.

A presença de exantema ocorre de dois a cinco dias após o início da febre em quase metade dos pacientes. 0 exantema é do tipo maculopapular, presente frequentemente em tronco e nas extremidades, mas pode ser verificado também nas regiões facial, palmar e plantar ${ }^{(5)}$. A presença do exantema maculopapular não foi expressiva no grupo de idosos estudados, considerando que a literatura relata que mais da metade dos indivíduos acometidos apresentam essa manifestação clínica ${ }^{(14-15)}$.

A caracterização de casos de febre de Chikungunya pode contribuir para identificar precocemente os casos suspeitos da doença para instituição de ações de cuidado à saúde e procedimentos de controle vetorial, oportunizados através de ações educativas e medidas de controle individual e coletivo de vetores. Indivíduos de qualquer idade são suscetíveis à infecção pelo CHIKV, no entanto, observa-se que o acometimento em idosos traz danos maiores. Essa condição se dá provavelmente pelo estado imunológico deprimido e pela associação a doenças preexistentes ${ }^{(15)}$.

Os resultados do estudo apresentam um maior contingente de idosos na fase crônica da doença, porém com uniformidade em relação à distribuição dos casos agudos e crônicos da enfermidade. A infecção possui evolução em três fases que são caracterizadas como aguda, subaguda e crônica. A fase aguda apresenta sintomas como febre alta, cefaleia e poliartralgia, podendo evoluir para a fase subaguda, com exacerbação da dor $\operatorname{articular}^{(12)}$. A doença pode, ainda, evoluir para uma fase crônica, com a presença de poliartralgia persistente ou recidivante com duração de semanas a anos, comprometendo as atividades de vida do paciente, reduzindo sua produtividade e causando importantes impactos econômicos na sua qualidade de vida ${ }^{(13)}$.

Algumas manifestações clínicas da fase crônica podem diversificar segundo sexo e idade. As mulheres são mais suscetíveis ao exantema, vômitos e sangramentos orais. Em pacientes mais idosos, a dor articular, edema e febre prologada são mais predominantes ${ }^{(5)}$. Esses dados também foram observados nos resultados da população estudada. A letalidade é maior no Brasil do que a constatada nas Américas, estando os casos graves e óbitos com presença mais elevada em pessoas com comorbidades e em extremos de idade. Tais fatores, somados aos apresentados neste estudo, destacam a relevância de intervenções de saúde preventivas e de promoção da saúde voltadas a essa população ${ }^{(15-17)}$.

A dor articular sem edema ou rigidez foi associada à maior probabilidade de recuperação. Alguns casos que evoluem para forma atípica da doença, com manifestações clínicas menos frequentes ou com maior gravidade, aparecem em torno de $0,3 \%$ da população de acometidos e estão ligados à idade avançada ( $>65$ anos) e às comorbidades associadas. Os casos mais graves estão também associados a relatos prévios de convulsão febril, diabetes mellitus, asma brônquica, doenças cardíacas e reumatológicas e hipertensão arterial sistêmica ${ }^{(2,15)}$. A população estudada está enquadrada nos critérios supraditos. Esses idosos podem estar em estado de vulnerabilidade para as formas mais graves da infecção pelo CHIKV.

Estudos de caracterização dos aspectos clínicos e epidemiológicos de doenças, em especial de agravos emergentes, são primordiais para o planejamento das ações de cuidado à saúde. Nos serviços de Atenção Primária à Saúde, os enfermeiros são reconhecidos como um profissional capaz de promover um cuidado integral e humanizado promovendo a educação em saúde e estabelecendo vínculos ${ }^{(16)}$. Nessa perspectiva, 
os resultados deste estudo podem colaborar para o cuidado individual e coletivo com base epidemiológica.

Assim como outras doenças crônicas, os sintomas persistentes associados à febre de Chikungunya são incapacitantes em termos de atividades da vida diária e prejudicam a qualidade de vida dos idosos. Embora a literatura mostre que os sintomas de longo prazo associados à Chikungunya estão presentes em todas as idades e em ambos os sexos, alguns grupos com pacientes idosos do sexo feminino com comorbidades subjacentes parecem estar em maior risco do que outros ${ }^{(17-19)}$.

Por se tratar de uma doença de circulação recente que tende a se espalhar para novas áreas, a febre de Chikungunya pode se tornar uma importante causa de desconforto crônico, dor musculoesquelética, artralgia, fadiga crônica e depressão, em especial na população idosa. Os profissionais de saúde devem estar preparadas para tratar pessoas com os sintomas da fase aguda e crônica da doença, abordando adequadamente as necessidades físicas, psicológicas e sociais ${ }^{(5,17-19)}$.

\section{Conclusão}

Os casos de infecção por CHIKV tendem a ter maiores repercussões clínicas em idosos, uma vez que os mesmos em sua maioria tiveram a necessidade de internação. Os sintomas identificados com maior frequência, neste estudo, foram a febre e a artralgia, seguidos de cefaleia e lombalgia.

\section{Colaborações}

Dourado CARO e Souza SRG contribuíram com a elaboração do projeto, análise de dados, redação, revisão crítica e aprovação final do artigo. Quirino EMB, Pinho CM e Silva MAS colaboraram com a redação, revisão crítica e aprovação da versão final. Andrade MS cooperou na análise dos dados, redação, revisão crítica e aprovação final.

\section{Referências}

1. Silva NM, Teixeira RAG, Cardoso CG, Siqueira Junior JB, Coelho GE, Oliveira ESF. Chikungunya surveillance in Brazil: challenges in the context of Public Health. Epidemiol Serv Saúde. 2018; 27(3):e2017127. doi: https://doi.org/10.5123/ S1679-49742018000300003

2. Ministério da Saúde (BR). Secretaria de Vigilância em Saúde. Monitoramento dos casos de dengue, febre de Chikungunya e a doença aguda pelo vírus Zika até semana epidemiológica 49 [Internet]. 2018 [citado 2019 abr 30]. Disponível em: http:// portalarquivos2.saude.gov.br/images/pdf/2019/ janeiro/02/2018-067.pdf

3. Castro AP, Lima RA, Nascimento JS. Chikungunya: vision of the pain clinician. Rev Dor [Internet]. 2016 [citado 2019 abr 30]; 17(4):299-302. Disponível em: http://www.scielo.br/scielo.php?script=sci_ arttext\&pid=S1806-00132016000400299

4. Amaral JK, Taylor PC, Teixeira MM, Morrison TE, Schoen RT. The Clinical features, pathogenesis and methotrexate therapy of chronic Chikungunya arthritis. Viruses. 2019; 11(3):289. doi: https:// doi.org/10.3390/v11030289

5. Van Aalst M, Nelen CM, Goorhuis A, Stijnis C, Grobusch MP. Long-term sequelae of chikungunya virus disease: A systematic review. Travel Med Infec Dis. 2017; 15:8-22. doi: http://dx.doi. org/10.1016/j.tmaid.2017.01.004

6. Masri $\mathrm{H}$. Chikungunya virus in the geriatric patient: an imported case. JAMDA. 2015; 16(3):6. doi: doi.org/10.1016/j.jamda.2015.01.011

7. Reis CB, Jesus RS, Silva CSO, Pinho L. Health conditions of young and old elderly. Rev Rene. 2016; 17(1):120-7. doi: http://dx.doi. org/10.15253/2175-6783.2016000100016

8. Milte CM, Walker R, Luszcz MA, Lancsar E, Kaambwa B, Ratcliffe J. How important is health status in defining quality of life for older people? An exploratory study of the views of older South Australians. Appl Health Econ Health Policy. 2014; 12(1):73-84. doi: http://dx.doi.org/10.1007/ s40258-013-0068-3 
9. Almeida AV, Mafra SCT, Silva EP, Kanso SA. Feminização da Velhice: em foco as características socioeconômicas, pessoais e familiares das idosas e o risco social. Textos Contextos. 2015; 14(1):115-31. doi: dx.doi.org/10.15448/16779509.2015.1.19830

10. Fiorati RC, Arcêncio RA, Souza LB. Social inequalities and access to health: challenges for society and the nursing field. Rev Latino-Am Enfermagem. 2016; 24:e2687. doi: https://dx.doi. org/10.1590/1518-8345.0945.2687

11. Lesser J, Kitron U. A geografia social do zika no Brasil. Rev Estud Av. 2016; 30(88):167-75. doi: dx.doi.org/10.1590/s0103-40142016.30880012

12. Azevedo RSS, Oliveira CS, Vasconcelos PFC. Chikungunya risk for Brazil. Rev Saúde Pública. 2015; 49(58):1-6. doi: https://doi.org/10.1590/ S0034-8910.2015049006219

13. Honório NA, Câmara DCP, Calvet GA, Brasil P. Chikungunya: an arbovirus infection in the process of establishment and expansion in Brazil. Cad Saúde Pública. 2015; 31(5):906-08. doi: http://dx.doi.org/10.1590/0102-311XPE020515

14. Marques CDL, Duarte ALBP, Ranzolin A, Dantas AT, Cavalcanti NG, Gonçalves RSG, et al. Recommendations of the Brazilian Society of Rheumatology for diagnosis and treatment of Chikungunya fever. Part 1 - Diagnosis and special situations. Rev Bras Reumatol. 2017; 57(2):421-37. doi: http://dx.doi.org/10.1016/j. rbre.2017.05.006
15. Ministério da Saúde (BR). Secretaria de Vigilância em Saúde. Febre Chikungunya: manejo clínico [Internet]. 2017 [citado 2019 abr 30]. Disponível em: http://bvsms.saude.gov.br/bvs/publicacoes/ febre_chikungunya_manejo_clinico.pdf

16. Souza KMJ, Seixas CT, David HMSL, Costa AQ. Contributions of public health to nursing practice. Rev Bras Enferm. 2017; 70(3):543-49. doi: http:// dx.doi.org/10.1590/0034-7167-2016-0401.

17. Paixão ES, Rodrigues LC, Costa MCN, Itaparica M, Barreto F, Gérardin P, et al. Chikungunya chronic disease: a systematic review and metaanalysis. Trans R Trop Med Hyg. 2018; 112(7):30116. doi: https://doi.org/10.1093/trstmh/try063

18. Duvignaud A, Fianu A, Bertolotti A, Jaubert J, Michault A, Poubeau $P$, et al. Rheumatism and chronic fatigue, the two facets of post-chikungunya disease: the TELECHIK cohort study. Epidemiol Infect. 2018; 146(5):633-41 doi: http://dx.doi. org/10.1017/S0950268818000031

19. Marimoutou C, Ferraro J, Javelle E, Deparis X, Somin F. Chikungunya infection: selfreported rheumatic morbidity and impaired quality of life persist 6 years later. Clin Microbiol Infect. 2015; 21(7):688-93. doi: http://dx.doi.org/10.1016/j. cmi.2015.02.024 